\title{
PROPIEDADES FÍSICO-MECÁNICAS Y TIPO DE FALLA ADHESIVA DEL ESMALTE HUMANO Y BOVINO
}

\author{
${ }^{1}$ Mauricio Benítez Díaz, ${ }^{1}$ Araxi Mirzoyan Kalach, ${ }^{2}$ Jaime Rodrigo Rivera Barrero, \\ ${ }^{3}$ Eliana Midori Tanaka Lozano \\ ${ }^{1}$ Estudiante Especialización en Ortodoncia Fundación CIEO, U. Militar Nueva Granada, Colombia. \\ ${ }_{2}^{2}$ Magíster en Ciencias Biomateriales, University of Alabama, Birmingham (EE. UU), profesor asociado Fundación CIEO, U. Militar Nueva Granada, Colombia. \\ ${ }^{3}$ Especialista en Ortodoncia Fundación CIEO, U. Militar Nueva Granada, Doctorado (PhD), profesora asociada Fundación CIEO, U. Militar Nueva Granada, Colombia.
}

\section{Autor responsable de correspondencia: Jaime Rodrigo Rivera B.}

Correo electrónico: jr.rivera@cieo.edu.co

\begin{abstract}
RESUMEN
Objetivo: comparar la fuerza de adhesión y tipo de falla entre el esmalte humano y bovino mediante el dispositivo universal de pruebas y estereomicroscopía.

Materiales y métodos: se realizó un estudio experimental In vitro para lo cual se utilizaron sesenta dientes, treinta primeros premolares superiores humanos y treinta incisivos inferiores bovinos en los cuales se cementaron brackets estándar metálicos (Ortho Organizers, California, EE. UU.) con resina compuesta (Transbond ${ }^{\circledR}$ XT, 3M Unitek, St. Paul, Minnesota, EE. UU.). Posteriormente, fueron descementados usando un dispositivo universal de pruebas (Instron 3367 Class 0,5 with load cell 0-30Kn, EE. UU.) a una velocidad de 1,5 mm/ min. Los datos fueron calculados en megapascales al dividir la fuerza por el área de la base del bracket. Para determinar el tipo de falla, la superficie de los dientes y las bases de los brackets fueron analizados mediante inspección visual con un estereomicroscopio (Stemi 2000C, Carl Zeiss, Göttingen, Germany).

Resultados: la fuerza de adhesión en dientes bovinos fue mayor al compararla con los dientes humanos (12,11 MPa y 6,10 Mpa, respectivamente) sin presentarse una diferencia estadísticamente significativa $(\mathrm{p}=0,052)$. Los tipos de falla predominantes para humanos y bovinos se presentaron en la interfase Resina/Bracket (RB 60,71\%) y Adhesivo/Esmalte (AE 57,14\%). El análisis de correspondencia múltiple reportó que a cualquier valor de fuerza se puede presentar cualquier tipo de falla en ambos dientes.

Conclusión: aunque no se encontró una diferencia significativa entre los grupos, no es concluyente la utilización de los dientes bovinos como sustitutos de dientes humanos en estudios de adhesión a esmalte según el método utilizado en esta investigación. [Benitez M, Mirxoyan A, Rivera JR, Tanaka EM. Propiedades físico-mecánicas y tipo de falla adhesiva del esmalte humano y bovino. Ustasalud. 2014; 13: 11 - 17]
\end{abstract}

Palabras clave: ortodoncia, esmalte dental, diente premolar.

\section{PHYSICAL AND MECHANICAL PROPERTIES AND FAILURE MODE COMPARISON OF HUMAN AND BOVINE ENAMEL}

\begin{abstract}
Objective: to compare the bond strength and failure mode between human and bovine enamel using the universal testing device and stereomicroscopy.

Methods: sixty teeth were used, thirty human upper premolars and thirty lower bovine incisor which were cemented with metallic standard brackets (Ortho Organizers, California, EE. UU.) using composite resin (Transbond ${ }^{\circledR}$ XT, 3M Unitek, St. Paul, Minnesota, EE. UU.) for later testing adhesion using the universal testing device (Instron 3367 Class 0.5 with load cell 0-30Kn, EE. UU.) at a speed of $1.5 \mathrm{~mm} / \mathrm{min}$. The data was calculated in megapascals, dividing the force by the area of the bracket base. Finally, in order to determine the failure mode, the tooth surfaces and the bases of the brackets, were analyzed by visual inspection using a stereomicroscope (Stemi 2000C, Carl Zeiss, Göttingen, Germany).

Results: the bond strength in bovine teeth was higher than in human teeth (12.11 MPa and 6.10 MPa, respectively) without an statistically significant difference $(p=0.052)$. The predominant failure modes for human and bovine teeth were presented at the interface Resin/Bracket (RB 60.71\%) and Adhesive/Enamel (AE 57.14\%). Multiple correspondence analyses reported that any force value could have any failure mode in both types of teeth.

Conclusion: although no statistically significant difference between groups was found, the applied method cannot determine whether it is possible the use of bovine teeth as a substitute for human teeth enamel in adhesion studies.
\end{abstract}

Key words: orthodontics, dental enamel, tooth, orthodontic brackets.

Recibido para publicación: 7 de febrero de 2014. Aceptado para publicación: 20 de mayo de 2014. 


\section{INTRODUCCIÓN}

Una de las mayores complicaciones para realizar estudios de investigación en ortodoncia y odontología general es la consecución de la muestra en cuanto a su tamaño y características ideales. A esto se suma que, desde hace unos años, los tratamientos odontológicos se han enfocado en la conservación de los tejidos dentales, lo que dificulta la obtención de especímenes para su realización. Hoy en día, en el área de la ortodoncia es indispensable realizar estudios para evaluar la fuerza de descementación de los brackets y el tipo de falla que estos presentan, puesto que existe mayor número de materiales, diseños, técnicas y protocolos que deben ser valorados para su uso en la práctica clínica. ${ }^{1,2}$

Por esta razón, es necesario utilizar sustitutos de dientes humanos. Gracias a su fácil consecución, uno de los sustitutos de mayor elección son los dientes bovinos; en algunas investigaciones se ha referenciado la similitud entre estos y los dientes humanos en cuanto a anatomía y composición histológica, pero esto carece de soporte y evidencia científica suficiente para ser usados como sustitutos en odontología..$^{3-6}$

Fowler y colaboradores en 1992 estudiaron la influencia de tres variables en la fuerza de adhesión y el tipo de falla de dos materiales (Scotchbond 2 y Ketac-Fil): modo de prueba (corte y tensión), diseño de dispositivos (tensión) y sustrato dentario (humano y bovino). Se concluyó que no existía una diferencia estadísticamente significativa en la fuerza de adhesión para los dos tipos de adhesivos. El tipo de falla para el adhesivo Scotchbond 2 reportó una diferencia estadísticamente significativa en tensión al comparar los dientes humanos (cohesiva) y los bovinos (adhesiva). Así mismo, en las pruebas de corte la mayoría de los especímenes bovinos presentaron fallas adhesivas mientras que los especímenes humanos no presentaron este tipo de falla. ${ }^{7}$

Oesterle y colaboradores también compararon la fuerza de adhesión en dientes humanos (incisivos permanentes) y bovinos (incisivos permanentes y deciduos), y reportaron una diferencia estadísticamente significativa entre los tres grupos, siendo los dientes humanos los que presentaron mayores valores de adhesión. ${ }^{8}$

Otros estudios han comparado el patrón de desgaste en esmalte humano y bovino, y el grado de erosión presente en ambos tejidos; se ha concluido que en dientes bovinos el desgaste fue mayor y estos se erosionaban más rápidamente que el diente humano. ${ }^{9,10}$ De otra parte, Posada y colaboradores en el 2006, en su revisión de la literatura, encontraron similitudes macroscópicas entre la dentadura humana y bovina. ${ }^{5}$

Desde la década de los ochenta hasta la actualidad, se han realizado diversos estudios que utilizan dientes bovinos; ${ }^{3-6}$ sin embargo, la evidencia científica no es suficiente con relación a si estos especímenes pueden sustituir de forma confiable a los dientes humanos.

Por tanto, el objetivo de esta investigación fue comparar la fuerza de adhesión y tipo de falla entre el esmalte humano y bovino mediante el dispositivo universal de pruebas y la estereomicroscopía.

\section{MATERIALES Y MÉTODOS}

Se realizó una investigación experimental In vitro con treinta primeros premolares superiores humanos extraídos con fines ortodóncicos y treinta incisivos inferiores bovinos. Para determinar el tamaño de la muestra se utilizó la fórmula de varianza mancomunada. Los premolares fueron recolectados en diferentes clínicas de la ciudad de Bogotá mientras que los incisivos inferiores bovinos provenían de animales sacrificados para el consumo humano. De este modo, por medio de inspección visual se incluyeron premolares e incisivos sanos que no presentaron alteraciones en el esmalte y se excluyeron aquellos con caries dental, fracturas y restauraciones en las caras vestibulares.

Inicialmente, los premolares (humanos) y los incisivos (bovinos) fueron limpiados por medio de curetaje para remover restos de tejido, y se sometieron a un proceso de desinfección con hipoclorito de sodio al 0,5\% para luego ser preservados por un máximo de tres meses en una solución de Cloramina $\mathrm{T}$ a temperatura ambiente. Para facilitar la manipulación de los especímenes, las raíces se embebieron en un cubo $(3 \times 1 \times 1 \mathrm{~cm})$ de resina acrílica.

Previo a la cementación de los brackets, la superficie vestibular de los dientes de ambos grupos fue sometida a una profilaxis con pieza de mano de baja velocidad, cepillo, agua destilada y bicarbonato. Posteriormente, los especímenes se lavaron con agua destilada por treinta segundos y se secaron con aire a presión durante diez segundos.

\section{Protocolos de cementación}

El protocolo de cementación utilizado fue el recomendado por el fabricante. Se realizó un grabado total con ácido fosfórico al 35\% (ScotchbondTM Phosphoric Etchant, 3M ESPE, St. Paul, Minnesota, USA) por quince segundos, se lavó con agua destilada por treinta segundos y se secó con aire a presión; luego se aplicó una capa de adhesivo en las 
superficies vestibulares, posteriormente se cargaron las bases de los brackets con resina (Transbond ${ }^{\circledR}$ XT, 3M Unitek, St. Paul, Minnesota, EE. UU.) y se procedió a realizar la ubicación de cada bracket (Estándar, Ortho Organizers, California, EE. UU.) en el centro de la corona clínica del diente mediante un porta bracket; se ejerció presión y se retiraron excesos con explorador, se polimerizaron por veinte segundos con la lámpara tipo LED (Optilight Max, GNATUS, Sao Paulo, Brasil).

\section{Pruebas mecánicas}

Para determinar la fuerza adhesiva se utilizó el dispositivo universal de pruebas (Instron 3367 clase 0,5 con celda de carga $0-30 \mathrm{Kn}$, EE. UU.) ubicado en la Universidad de los Andes. La máquina consta de una cruceta que ejerce una fuerza de corte sobre la interfase esmalte-bracket y unas mordazas neumáticas que sujetan el espécimen. La velocidad de aplicación de la fuerza fue de $1,5 \mathrm{~mm} / \mathrm{min}$, en dirección ocluso-gingival hasta producir el desprendimiento de los brackets (Figura 1).
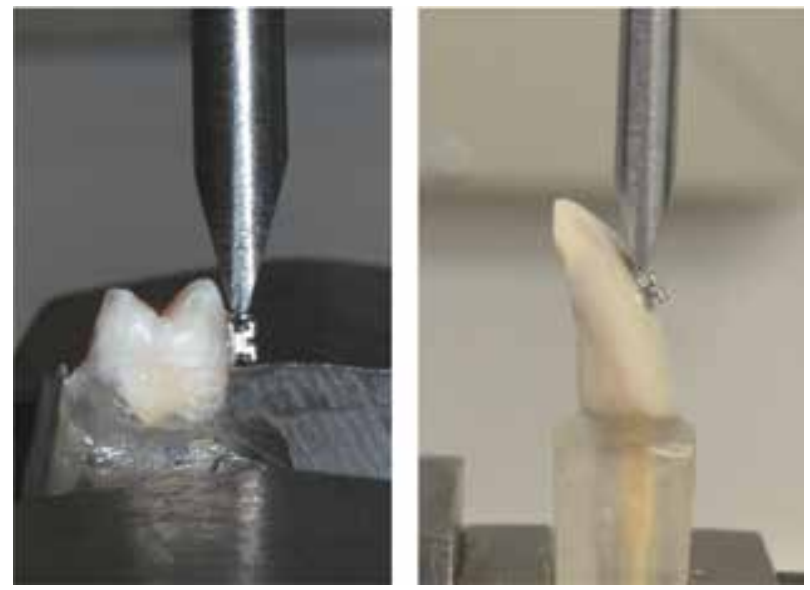

Figura 1. Prueba mecánica para la descementación mediante dispositivo universal de pruebas. En la imagen de la izquierda se observa el diente humano y en la derecha, el diente bovino

\section{Estereomicroscopía}

Una vez descementados los brackets, se determinó el tipo de falla. Se observaron las superficies de los dientes y las bases de los brackets con un estereomicroscopio (Stemi 2000C, Carl Zeiss, Göttingen, Germany) al que se encuentra conectado la AXIOCAM (Carl Zeiss Microimaging GmbH 37081, Göttingen, Germany) ubicada en la Fundación CIEO, con una magnificación de 50X. Para establecer el tipo de falla observado, se usó la clasificación propuesta por el Medical College of Georgia: ${ }^{11}$

* RB: resina-bracket (adhesiva)

* RR: resina-resina (cohesiva)

* AE: adhesivo-esmalte (adhesiva)

* EE: esmalte-esmalte (cohesiva)

* ED: esmalte-dentina (adhesiva)

\section{Análisis estadístico}

Inicialmente, se realizó un análisis de los puntos atípicos por medio de un box plot y se determinó excluir dos datos para cada grupo. Luego se realizó la prueba de Shapiro-Wilk que determinó que ninguno de los grupos presentaba una distribución normal, por lo cual se aplicó la prueba de Levene que arrojó una diferencia estadísticamente significativa en la homogeneidad de las varianzas de las medianas de la fuerza adhesiva, razón por la cual se aplicó la prueba de Mann Whitney para determinar si existía una diferencia estadísticamente significativa en la fuerza de adhesión entre los grupos.

La prueba $Z$ de proporciones determinó si también existía una diferencia significativa entre los tipos de falla y luego la prueba exacta de Fisher estimó si existía una asociación significativa entre el tipo de falla y el tipo de diente. Finalmente, se aplicó un análisis de correspondencia múltiple entre la fuerza, el diente y el tipo de falla.

\section{RESULTADOS}

\section{Fuerza adhesiva}

Las medianas de fuerza adhesiva para los dos grupos no presentaron una diferencia estadísticamente significativa $(p=0,052)$ con valores de $6,10 \mathrm{MPa}$ y 12,11 MPa para los dientes humanos y bovinos, respectivamente (Figura 2).

\section{Tipo de falla}

Para cuantificar los datos del tipo de falla se realizó un análisis porcentual, tanto para el grupo de los dientes humanos como para el grupo de los dientes bovinos. Es importante anotar que para ninguno de los grupos se presentó el tipo de falla EE y para el grupo de dientes bovinos tampoco se presentó el tipo de falla ED.

Como se observa en la Figura 3, al evaluar el tipo de falla para los dientes humanos se encontró que el mayor porcentaje fue el tipo $\mathbf{R B}$, seguido por $\mathbf{R R}$, luego $\mathbf{A E}$ y por último ED; el tipo de falla correspondiente a EE no se presentó en ningún espécimen. Así mismo, las figuras 4 a, b, c y d, muestran microfotografias de las diferentes fallas presentadas en dientes humanos.

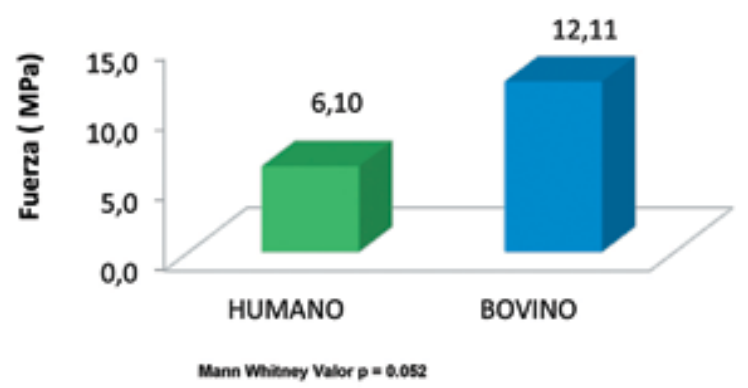

Figura 2. Comparación de la mediana entre los grupos evaluados 


\section{ARTÍCULO DE INVESTIGACIÓN CIENTÎFICA Y TECNOLÓGICA}

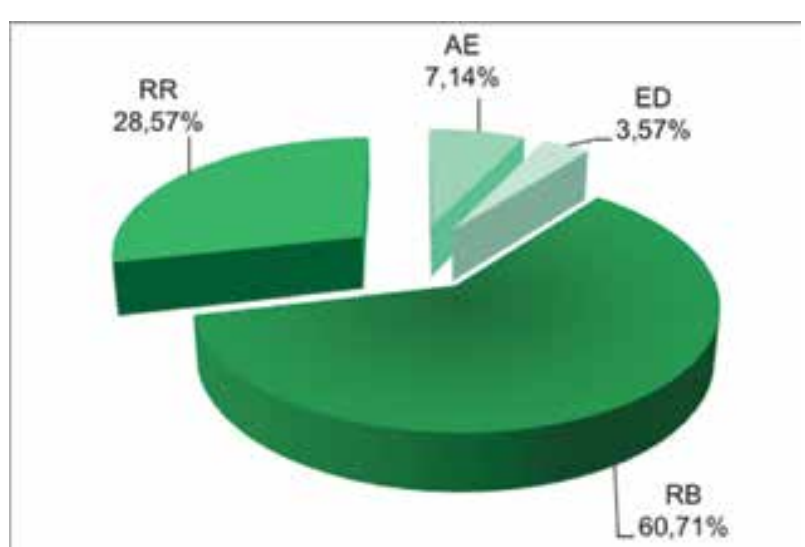

Figura 3. Distribución porcentual del tipo de falla en los dientes humanos

a.
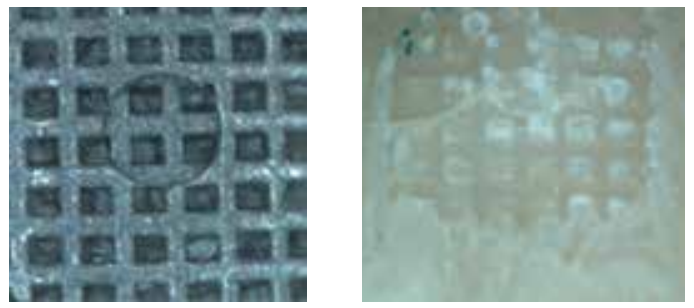

b.
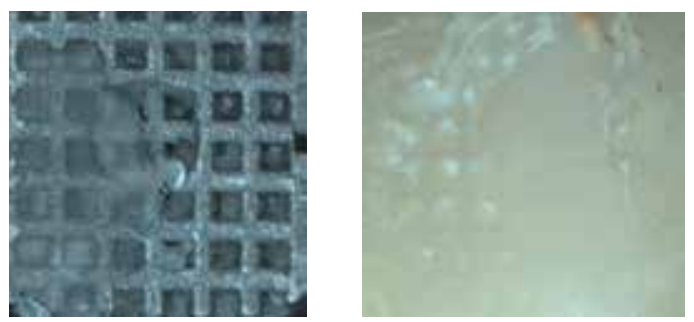

c.

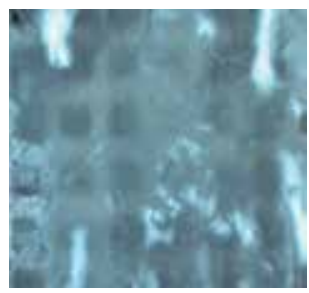

d.
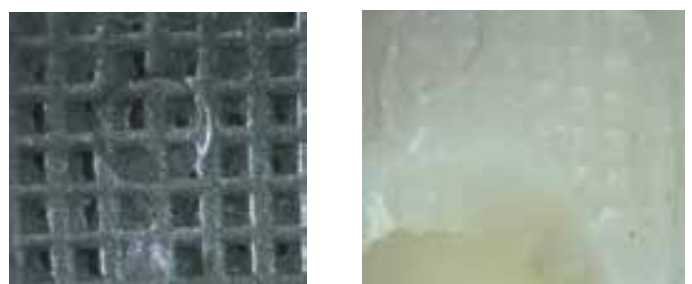

Figura 4. Falla en la interfase: a. Resina/Bracket (RB), b. Resina/Resina (RR), c. Adhesivo/Esmalte (AE), d. Esmalte/Dentina (ED) en dientes humanos

Por el contrario, para los dientes bovinos (Figura 5), el tipo de falla que se presentó con mayor frecuencia fue $\mathbf{A E}$, seguido por RR y por último RB; los tipos de falla correspondientes a ED y EE no se presentaron en ningún espécimen. En las figuras $6 \mathrm{a}, \mathrm{b}, \mathrm{y} \mathrm{c}$, se observan microfotografias de las diferentes fallas presentadas en dientes bovinos.

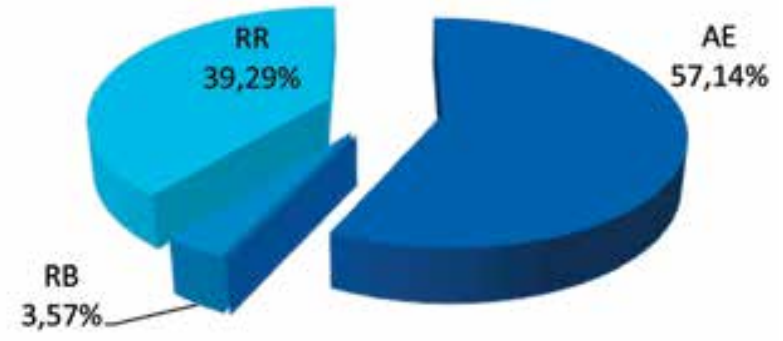

Figura 5. Distribución porcentual del tipo de falla en dientes bovinos

a.

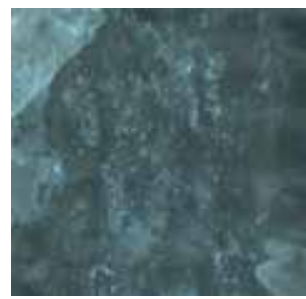

b.

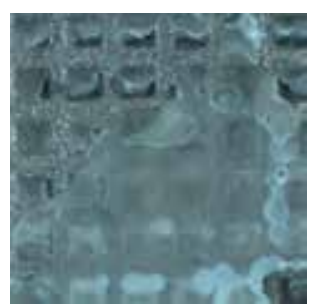

c.
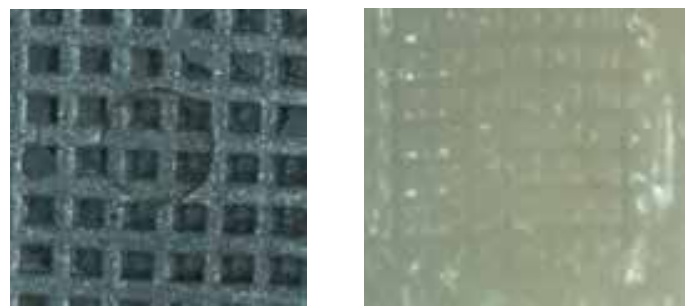

Figura 6. Falla en la interfase: a. Adhesivo/Esmalte (AE), b. Resina/Resina (RR), c. Resina/Bracket (RB) en dientes bovinos

La prueba exacta de Fisher determinó una asociación estadísticamente significativa entre el tipo de falla y el tipo de diente $\left(p=6,84 \mathrm{e}^{-07}\right)$. Los dientes humanos presentaron una asociación con tipos de falla $\mathbf{R B}$ y $\mathbf{R} \mathbf{R}$ y los dientes bovinos fueron asociados con tipos de falla $\mathbf{A E}$ y $\mathbf{R R}$.

Por último, se realizó un análisis de correspondencia múltiple por medio de la prueba de $\mathrm{Chi}^{2}$ la cual reportó que a cualquier magnitud de fuerza se puede presentar cualquiera de los tipos de falla encontrados y esto puede suceder tanto en los dientes humanos como en los bovinos. Sin embargo, se constató una tendencia de asociación entre los dientes humanos y los tipos de falla $\mathbf{R B}$ y RR, con un valor de fuerza esperado de 6,10 MPa mientras que los dientes bovinos se asociaron a los tipos de falla $\mathbf{A E}$ y $\mathbf{R R}$, con un valor de fuerza esperado de 12,11 MPa (Figura 7). 


\section{AN ÁLISIS DE CORRE PONDENCIA MÚLTIPLE FUERZA ADHE SIVA-DIENTE-TIPO FALLA}

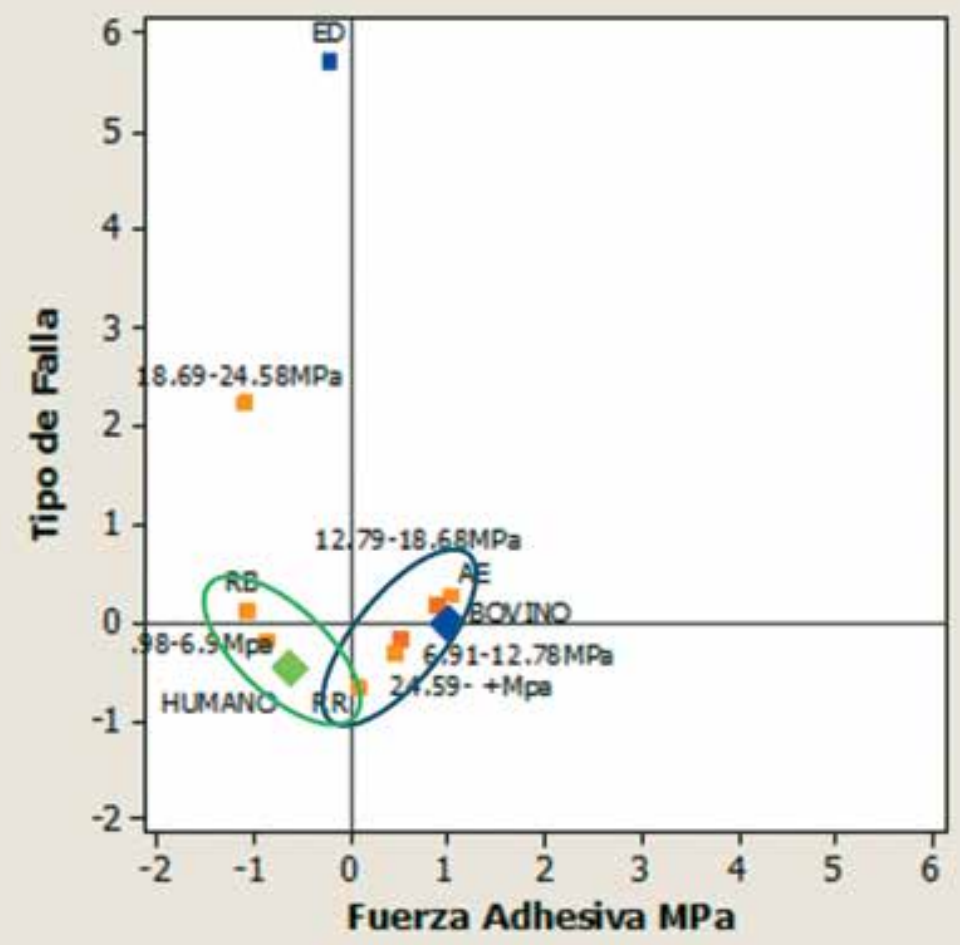

Figura 7. Análisis de correspondencia múltiple

\section{DISCUSIÓN}

\section{Fuerza adhesiva}

En este estudio se encontró que no existe una diferencia estadísticamente significativa en la fuerza de adhesión entre los dientes humanos y los dientes bovinos. Sin embargo, es importante resaltar que en este estudio, la fuerza presentada en los dientes bovinos (12,11 Mpa) correspondió al doble de la fuerza presentada en los dientes humanos (6,10 MPa).

Adicionalmente, se observó que las fuerzas obtenidas tanto en dientes humanos como bovinos son más bajas comparadas con lo reportado en la literatura. ${ }^{12-14}$ En este estudio debido a que se presentó una mayor área de contacto entre la cruceta del dispositivo universal de pruebas y el bracket, la fuerza no se aplicó sobre la base de esta, sino entre la base y las aletas, lo cual disminuyó la carga necesaria para su desprendimiento según lo reportado previamente por Klocke y colaboradores, quienes afirmaron que cuando se aplica la fuerza sobre la base del bracket, se necesita de mayor fuerza para la descementación, al igual que cuando se aplica la fuerza paralela a la base de este. ${ }^{15}$

Klocke y colaboradores confirmaron esta afirmación al realizar un estudio con 150 dientes bovinos a los que se les aplicaron fuerzas paralelas a la base de los brackets y con diferentes grados de inclinación, encontraron que los brackets que fueron descementados con una desviación de $15^{\circ}$ obtuvieron una fuerza de 22,99 Mpa, contrario a los especímenes que fueron descementados con una fuerza paralela a la base del bracket, los cuales obtuvieron fuerzas menores (17,90 MPa). ${ }^{16}$ A pesar de que en el presente estudio se observaron las medianas de las fuerzas, está comprobado que a mayor resistencia a la angulación de la fuerza de resistencia, mayor fuerza adhesiva.

Al revisar la literatura se encuentran estudios como el de Sfondrini y colaboradores donde en una muestra de cuarenta dientes bovinos, ${ }^{14}$ se obtuvieron medianas de fuerzas mayores a las encontradas en el presente trabajo. Estos brackets fueron descementados con un dispositivo universal de pruebas que presentaba una punta plana de menor tamaño y una orientación paralela a su misma base, obteniendo una mediana de 22,8 MPa.

Por otro lado, al comparar nuestros resultados con lo reportado previamente por Vilar y colaboradores, ${ }^{17}$ los datos para la fuerza adhesiva son similares (11,22 MPa). No obstante, se debe aclarar que en este estudio se reportan valores en medianas debido a que los datos no fueron paramétricos, contrario a los datos de Vilar y colaboradores que fueron paramétricos. 
Así mismo, Fernández y colaboradores en 1999 compararon la fuerza de adhesión de dos brackets estéticos con un grupo control de brackets metálicos en dientes bovinos y obtuvieron un promedio de 13,21 MPa para los especímenes cementados con brackets metálicos..$^{18}$ Sin embargo, el tipo de fuerza utilizado en su estudio fue una fuerza tensional, es decir, se traccionaron los brackets, por lo cual se puede asumir que el bracket al ser traccionado necesita menor fuerza para su desprendimiento.

Con respecto a los dientes humanos, la fuerza adhesiva obtenida en este estudio fue de 6,10 MPa que puede considerarse como baja al compararla con estudios In vitro; así pues Ciceri y colaboradores obtuvieron medianas de fuerza mayores $(28,98 \mathrm{MPa})$ específicamente en grabado total al comparar dos sistemas adhesivos para ortodoncia. ${ }^{12}$ Se debe hacer referencia a que la cruceta utilizada en esta investigación presentaba una punta plana y de menor tamaño, lo cual, como ya se mencionó, facilita que la fuerza ejercida sea paralela a la base del bracket y lo más cerca posible a la superficie del esmalte, lo que podría aumentar la fuerza necesaria para su desprendimiento.

De igual manera, Polat y colaboradores verificaron las fuerzas de adhesión de tres grupos: dos grupos de cementado indirecto y un grupo de cementado directo, teniendo este último el promedio de fuerza de adhesión mayor (12,8 MPa). ${ }^{13}$ Una vez más en este estudio se utilizó una cruceta de punta plana lo cual favorece que la dirección de la fuerza sea paralela a la base del bracket.

Pickett y colaboradores realizaron una comparación In vitro e In vivo de fuerza de adhesión, al utilizar un dispositivo que medía la fuerza de adhesión al descementar los brackets intraoralmente, ${ }^{19}$ concluyeron que el grupo In vivo obtuvo fuerzas significativamente menores (5,47 MPa) que el grupo In vitro $(11,02 \mathrm{MPa})$ descementado con el dispositivo universal de pruebas. Pese a que estos datos son los promedios de las fuerzas, se podría concluir que por el gran tamaño de la cruceta utilizada en el presente estudio simuló el movimiento ejecutado en boca al momento de retirar los brackets por parte del profesional. El dispositivo universal de pruebas al ser estandarizado con una punta plana y un tamaño adecuado produce mayores fuerzas, pero si es reemplazado por una cruceta que abarca una mayor superficie del diente se presentan no solo fuerzas de cizallamiento sino una combinación de fuerzas tales como tensionales, cizallamiento y desprendimiento. Esto ocurrió al descementar los brackets con el dispositivo intraoral, puesto que la fuerza fue aplicada entre la base y las aletas de los brackets, al igual que en el presente estudio, lo que puede generar un movimiento rotacional y así disminuir la fuerza necesaria para descementar los brackets.

\section{Tipo de falla}

Se encontró que existe una diferencia estadísticamente significativa en relación con el tipo de falla presentada en ambos tipos de dientes. El tipo de falla predominante para los dientes bovinos fue en la interfase $\mathbf{A E}$ con un $57,14 \%$. Como ya se mencionó, la fuerza ejercida en la descementación de los brackets en el grupo bovino es el cizallamiento, dado que la cruceta utilizada era de gran tamaño y la dirección no era paralela a la interfase entre la base del bracket y el diente, esta ejerció un movimiento de palanca el cual se asume, desprende toda la resina del diente. Mientras que en los dientes humanos la falla que más prevaleció fue en la interfase RB con un $60,71 \%$, igualmente al presentarse una cruceta de gran tamaño la fuerza ejercida fue más hacia las aletas del bracket, lo cual facilitó el desprendimiento del bracket sin llevarse consigo la resina.

Otros estudios también muestran el mismo tipo de falla obtenida en el presente estudio para los dientes bovinos. A pesar de haber utilizado clasificaciones distintas, Vilar y colaboradores obtuvieron un tipo de falla ARI 0 (sin presencia de adhesivo en el diente) que corresponde al tipo de falla $\mathbf{A E} .{ }^{17}$ Así mismo, Fernández y colaboradores encontraron fallas de tipo ARI 2 (más de la mitad del adhesivo permanece en el diente) en dientes bovinos, que correspondió a fallas de tipo RR, con lo que se podría pensar que al ser traccionados los brackets se produce una falla cohesiva del material. ${ }^{18}$

De otro lado, Sfrondini y colaboradores en su investigación al utilizar la punta de la cruceta paralela a la base del bracket obtuvieron fuerzas de cizallamiento que hicieron que al desprenderse los brackets en los dientes bovinos, se presentara un tipo de falla ARI 3 (superficie del esmalte cubierta completamente por adhesivo), ${ }^{14}$ contrario a lo encontrado en la presente investigación.

Con respecto a los dientes humanos, como ya se mencionó, el tipo de falla de mayor prevalencia en el presente estudio fue $\mathbf{R B}$. Al revisar la literatura se encontró que algunos estudios realizados en dientes humanos presentaban fallas de tipo $\mathbf{A E},{ }^{12}$ o fallas ARI 5 (sin remanente de resina en el diente). ${ }^{13}$ En ambos estudios se utilizó una cruceta de punta plana de menor tamaño, de manera que la fuerza fue ejercida sobre la base de los brackets y no entre la base y las aletas, lo que favorece que no exista remanente de resina en el diente al momento de la descementación. 


\section{ARTÍCULO DE INVESTIGACIÓN CIENTÎFICA Y TECNOLÓGICA}

Por tanto, se puede concluir que a pesar de no encontrar una diferencia estadísticamente significativa entre los grupos, el método aplicado en la presente investigación no permite determinar si es posible el uso de los dientes bovinos como sustitutos de los dientes humanos en estudios de adhesión a esmalte. Es necesario realizar la estandarización del método al aplicar las pruebas de adhesión en dientes humanos y bovinos al tener en cuenta la inclinación de los dientes, punto de aplicación de la fuerza, el tamaño y la forma de la cruceta utilizada, variables que pueden influir en el resultado.

\section{BIBLIOGRAFÍA}

1. Ozturk B, Malkoc S, Koyuturk EA, Catalbas B, Ozer F. Influence of different tooth types on the bond strength of two orthodontic adhesive systems. Eur J Orthod. 2008; 30: 407-412.

2. Lunardi N, Gameiro H, Borges M, Nouer D, Vieira V, Consani S, Sarmento J. The effect of repeated bracket recycling on the shear bond strength of different orthodontic adhesives. Braz J Oral Sci. 2008; 7: 1648-1652.

3. Nkenke E, Hirschfelder U, Martus F, Eberhard H. Evaluation of the bond strength of different bracket-bonding systems to bovine enamel. Eur J Orthod. 1997; 19: 259270.

4. Oshiro J, Medici E, Pereira J, Castillo M. Comparative analysis of human and bovine teeth: radiographic density. Braz Oral Res. 2008; 22: 346-351.

5. Posada M, Sánchez C, Gallego G, Vargas A, Restrepo L, López J. Dientes de bovino como sustituto de dientes humanos para su uso en odontología. Revisión de la literatura. Rev CES Odontol. 2006; 19: 63-68.

6. Nakamichi I, Iwaku M, Fusayama T. Bovine teeth as possible substitute in adhesion test. J Dent Res. 1983; 62:10761083.

7. Fowler C, Swartz M, Moore B, Rhodes B. Influence of selected variables on adhesion testing. Dent Mater. 1992; 8: 265-269.

8. Oesterle L, Shellhart W, Belanger G. The use of bovine enamel in bonding studies. Am J Orthod Dentofacial Orthop. 1998; 113: 514-519.

9. Grassi L, Canhizares C, Barbosa R, Guaglianoni D, Pereira L. Human teeth versus bovine teeth: cutting effectiveness of diamond burs. Braz J Oral Sci. 2010; 9: 39-42.

10. White A, Yorath C. Human and bovine enamel erosion under single drink conditions. Eur J Oral Sci. 2010; 118: 604-609.

11. Georgia's Health Science University-School of Dentistry. Bond failure analysis. January 2003.

12. Ciceri A, Monroy J, Ardila G, Luna A, Rivera J. Comparación de la fuerza adhesiva y el tipo de falla de dos sistemas adhesivos para ortodoncia. Ustasalud. 2011; 10: 29-35.

13. Polat O, Karaman A, Buyukyilmaz T. In vitro evaluation of shear bond strengths and In vivo analysis of bond survival of indirect-bonding resins. Angle Orthod. 2004; 74: 405-409.

14. Sfondrini M, Scribante A, Cacciafesta V, Gandini P. Shear bond strength of deciduous and permanent bovine enamel. J Adhes Dent. 2011; 13: 227-230.
15. Klocke A, Kahl-Niekeb B. Influence of the force location in orthodontic shear bond strength testing. Dent Mater. 2005; 21: 391-396.

16. Klocke A, Kahl-Niekeb B. Effect of debonding force direction on orthodontic shear bond strength. Am J Orthod Dentofacial Orthop. 2006; 129: 261-265.

17. Vilar R, Souza N, Cal-Neto J, Galvao M, Sampaio H, Moraes A. Shear bond strength of brackets bonded with two light-curing orthodontic adhesives. J Adhes Dent. 2009; 11: 259-262.

18. Fernández L, Canut J. In vitro comparison of the retention capacity of new aesthetic brackets. Eur J Orthod. 1999; 11: 71-77.

19. Pickett K, Sadowsky L, Jacobson A, Lacefield W. Orthodontic in vivo bond strength: comparison with In vitro results. Angle Orthod. 2001; 71: 141-148.

\section{Correo electrónico de los autores:}

Mauricio Benítez Díaz: missobenitez@hotmail.com Araxi Mirzoyan Kalach: araxi.mirzovan@hotmail.com Jaime Rodrigo Rivera Barrero: jr.rivera@cieo.edu.co Eliana Midori Tanaka Lozano: emtanaka@hotmail.com 